\title{
INTEGRATED SURVEYNG WITH MOBILE MAPPING SYSTEM, EGNOS, NTRK AND LASER TECHNOLOGIES IN THE PARK “NINNI CASSARA” IN PALERMO
}

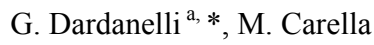 \\ ${ }^{\text {a }}$ University of Palermo, Italy, Dipartimento di Ingegneria Civile, Ambientale e Aerospaziale, \\ Viale delle Scienze, 90128 Palermo, Italy - \\ gino.dardanelli@unipa.it
}

Commission II, WG II/2

KEY WORDS: GNSS, RTK, NRTK, PERMANENT STATIONS, GLONASS, MMS, LASER SCANNER, 3DMODELLING, MULTI SENSOR INTEGRATION

\begin{abstract}
:
This article summarizes the experience gained between 2012 and 2013 by the department of "Civil Engineering, Environmental, Aerospace and Materials" of University of Palermo on the integrated survey of Ninni Park Cassara Park in Palermo and the subsequent testing of methods, tools and techniques based on current research regarding the acquisition and processing of GNSS (Global Navigation Satellite System) data and laser-scanner. A fruitful time dedicated to the design of the survey has allowed us to become aware of the critical issues that the site presents because of its vast extent and diversity in size and number of the elements of which it is composed. The work has been addressed thematizing the elements to detect and selecting the techniques as possible economic and fast to be applied in the acquisition phase. Sixteen control points evenly distributed within the site were first materialized and detected with static GNSS mode.

The survey mode NRTK (Network Real Time Kinematic) of the elements was then planned and carried out. The survey of the numerous planting was done by exploiting the mode with EGNOS (European Geostationary Navigation Overlay Service) correction. We continued the work experimenting with MMS (Mobile Mapping System) acquisition through which it was possible to acquire data on the morphology of the terrain, the conditions of the state of footpaths, buildings and on the distribution of street furniture. The point clouds obtained were subjected to both automatic and manual procedures to verify, finally, their actual descriptive possibilities of real forms detected.
\end{abstract}

\section{Introduction}

The park is the subject of study extends along a portion of agro Palermo, in the area south-east of the old core of the city. With a total area of over 28 hectares, spread from the Pit of Garofala (archaeological site), up to the present inner ring road of the city (the deviation of the bed of Kemonia to the Oreto), bordered on the west by the way Alton and the Corso Pisani, on the east by the University of Palermo. The old park, known as the "Garden of Orleans", was built around 1812 by Louis Philippe Orleans. At that time, with an area of about 66 acres, included a vast orchard and an area devoted to the cultivation of vegetables, along with some ornamental and recreational adjacent to the villa and a wider turned into a landscaped garden. Over time, because of the numerous changes of ownership, the entire area has undergone several transformations to its complete abandonment. Only between 1954 and 1957, the Sicilian Region has purchased the property entrusting the design and construction of the new park technical officials of the Town Planning of the City of Palermo.

The Fundamental, in the course of this work, was the first moment of reflection on the criticality of the site that has confirmed the need to have a well-structured design of the survey. This work has addressed, through a careful analysis of the problems of the site and available technologies to strategic decisions affecting the phase of acquisition and processing of data. Sixteen control points evenly distributed within the site have been materialized and detected with static GNSS mode to perform checks on the data acquired subsequently. We have chosen to use the MMS IPS2 provided by Topcon for the survey of the morphology of the soil, of the surrounding buildings, the urban design and the verification of the status conditions of the trails (NRTK acquired with Leica GS15 receiver). The acquisition of data concerning the countless species of trees on the site was then performed using the handheld Trimble GeoXH exploiting EGNOS corrections positioning mode.

The development of NRTK GNSS in the last years is probably due to the fact that these infrastructure has been proven to be an efficient technology for high accuracy positioning.

Today networks of permanent stations represent an innovation in the traditional mode of topographic survey, because solve one critical limitation of conventional RTK approach that is the distance between the reference and rover receivers. Many scientific studies have been conducted to determine the accuracy, the reliability and the repeatability of tests conducted in NRTK GNSS at universities, research institutions, public and private institutions.

One of the first studies was carried out in Canada, by Department of Geomatics Engineering, University of Calgary. The Multiple Reference Stations Approach (MultiRef) was used to understand the effects of network geometry on the achievable user accuracy. The tests show that the best performance is achieved when the reference stations are balanced on all sides 
of the rover receiver and that reference stations external to these core stations do not contribute to performance improvement (Lachapelle et al. 2003). All the international studies were conducted according to different methodologies and mathematical models for calculation of the network correction. The size, the number of stations and the geometric configurations change significantly and, furthermore, some tests have been conducted with long time static survey.

In this way EGNOS is inserted in a complementary manner in the global navigation systems dedicated to providing users with the accuracy of the positioning errors of position with latency does not exceed 6 seconds. With regard to experiences in recent years with EGNOS, they have confirmed the accuracies obtained are considered better than traditional navigational system, although still not to be involved in topographic survey. Oszczak et al. (2005) carried out experiences for the performance of the EGNOS system, especi ally in terms of the correction algorithms WADGPS (Wide Area Differential GPS) and position accuracy. The main lines of research have focused on MMS (Mobile Mapping Systems) in the 90's the study of algorithms on the determination of position and attitude of the vehicle and procedures on the data processing MMS in Canada through the scientific literature of Schwarz K.P. and El-Sheimy N. (1996), the theme of the cadastre roads that currently holds a role of primary importance by virtue of the enactment of Italian regulations of which we highlight the work of Siligato G. et al. (2004); environmental monitoring data integration with multibeam mapping of the coastal profile / backdrop, the generation of geographic databases compatible with GIS systems, the morphological survey of coastline, banks of rivers or lakes, cliffs and promontories, the suvey of structures such as dams, jetties, docks and port yards and, in general, the infrastructure and its appurtenances to which they are dedicated, for example, studies of Dondi G. et al. (2010), the "city modeling" which are dedicated to research carried out by Schwarz and El-Sheimy N. et al. (1996), the extraction of digital surface models (DSM) and the terrain (DTM) and bathymetric surveys carried out along the coast or river with profilers or scanner sonar as in studies of A. Adami et al. (2011).

\section{Main body}

Will be shown in chronological order software tools and results of all three approaches described as applied in the context of the integrated survey of N. Park Cassara in Palermo.

\subsection{NRTK survey}

We initially chose to recognize the pedestrian and cycle paths through the positioning system NRTK. Sixteen control points have nevertheless been materialized and measured in static mode and cataloged through monographs that illustrate the plane coordinates in the geographical systems WGS84 and Gauss- Boaga, the number of satellites available and the parameter DOP in the acquisition phase. The real-time correction was handled by the network of permanent stations of the University of Palermo, Dardanelli et al. (2009).

For the survey of the 16 control vertices an acquisition time of 60 seconds was set. The acquisition sessions were repeated at the beginning and at the end of each day's survey. The receiver has acquired the real-time corrections from the network of permanent stations of the University of Palermo through the protocol Ntrip using the package data connection. The acquisition time set for the prominence of the points of detail has been instead of 1 second. The raw data have allowed us to see a complete overview of 3786 measuring points (which is fixed and float). Acquiring data mode NRTK does not need any post-processing of the data, it was still controlled the level of precision achieved $(1-5 \mathrm{~cm})$. The points that have submitted deviations higher, especially those whose phase ambiguity has not been fixed (float), were eliminated from the calculation. For the reports of this work we have created five templates (WGS84 geographic coordinates, standard deviation, as in three dimensions, number of satellites and DOP indexes) that made possible the processing of the results. The framing of the survey in the digital mapping of the city of Palermo has made us need to work with plane coordinates Gauss-Boaga. For accuracy it is then preferred to export to a text file all the geographic coordinates and perform the transformation through the software Verto3k. This software has enabled us to convert the positions in geographic coordinates and plane coordinates (ROMA 40 ETRF89, ETRF2000). The final number of points used for drawing is 3056. For this reason, we used a tool designed to Autocad (ArkiTool) that lets you use a properly formatted text files in a single operation to insert the point cloud. The extracted data were first superimposed in CTR (Regional Technical Map) in digital format for verification. A first analysis of the results has been obtained through the analysis of the parameters DOP (Dilution of Precision). The accuracy with which the position is determined is in fact closely related to the error of the measurement by means of this factor that can be expressed as the ratio between the accuracy in the position and extent. The local test is performed on the single observation under the assumption of independent observations and the absence of model errors. This is a test of statistical analysis that determines whether observation $i$ is a value numerically distant from the rest of the collected data (outliers). In our work, the quality index $\mathrm{X}$ is generally in the range of the value 0.01-0.04 $\mathrm{m}$, presenting a high concentration in the neighborhood of $0.015 \mathrm{~m}$ (Fig. 1). It can be seen from the graph that $80 \%$ of surveyed points possesses quality $\mathrm{X}$ in the range $0.01-0.02 \mathrm{~m}, 12 \%$ in the range $0.02-0.03 \mathrm{~m}, 4 \%$ from $0,03 \mathrm{I}$ $0.04 \mathrm{~m}$, and only $3 \%$ over $4 \mathrm{~cm}$. The quality index Y lies in the range $0.03 \mathrm{~m} 0.005 \mathrm{~m}$, the trend line (Fig. 2) shows how this index is centered around the value of $1 \mathrm{~cm} .77 \%$ of the points is contained in the range subcentimeter of this index, $20 \%$ is between $0.01 \mathrm{ft} 0.02 \mathrm{~m}$, and $3 \%$ between 0.02 and $0.03 \mathrm{~m}$. Finally one can observe that the quality of the z-coordinate presents an interval of values from 0.01 to $0.05 \mathrm{~m}$, the trend line is almost constant at the value of $0,019 \mathrm{~m}$. The graph shows that $72 \%$ of the points has quality $\mathrm{Z}$ in the range $0.01-0.02 \mathrm{~m}, 21 \%$ had values between $0.02-0.03 \mathrm{~m}, 5 \%$ from 0.03 to $\mathrm{m} 0,04 \mathrm{~m}$ that $2 \%$ only shows values greater than $4 \mathrm{~cm}$. The scatter plot shows that the range of values of standard deviation is between $0.006-0.04 \mathrm{~m}$. The trend line is globally constant at $0,014 \mathrm{~m}$, the highest percentage of values is concentrated below it. The standard deviation of $\mathrm{Y}$ is in the range of values between $0.02 \mathrm{~m}$ $0.003 \mathrm{~m}$, the graph also shows a trend line constant in the neighborhood of $0.007 \mathrm{~m}$. The few points that fall outside of the above turn out to be the same, out of range, which are observed in the graphs of the other parameters already analyzed.

The standard deviation of $Z$ (Fig. 3), although it is concentrated at values lower than $0.01 \mathrm{~m}$ (this is the value at which you look at the trend line), has a more uniform distribution than that in the $\mathrm{X}$ direction and $\mathrm{Y}$ direction. 


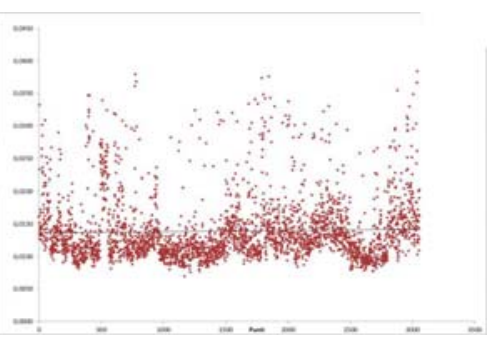

Fig.1: X Standard deviation of NRTK survey

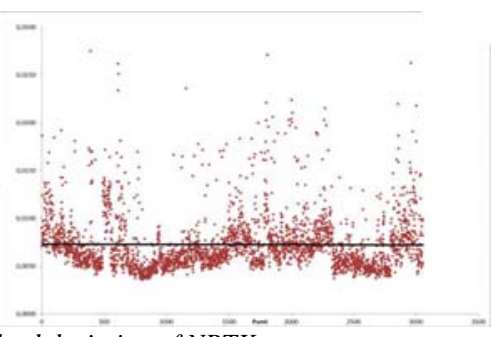

Fig.2: Y Standard deviation of NRTK survey

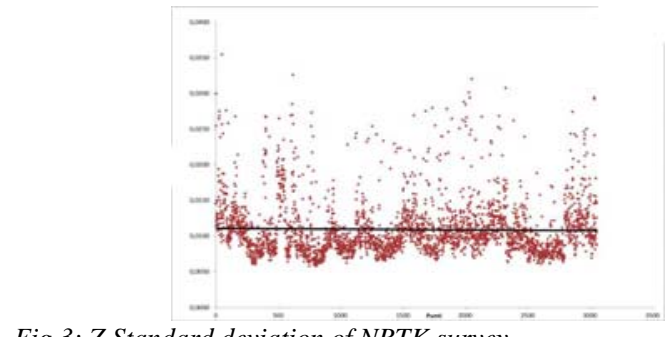

Fig.3: Z Standard deviation of NRTK survey

The last analysis performed is related to the number of satellites used for acquisitions. In order to obtain good accuracy is important to check the number of satellites and their geometric configuration, as has been mentioned earlier are the indices DOP to measure the accuracy degradation as a function of such a configuration as showed in literature by Grejner et al. an Aponte et al..

\subsection{EGNOS survey}

The purpose of this study was to verify the reliability of the system with respect to planimetric and altimetric accuracy of the individual points raised through the study and data processing (Cefalo et al.). The final stage of this work has focused on the post-processing of the raw data in the EGNOS correction mode. Survey operations have involved 4,124 entities, divided into 3 categories: Points, Lines and Areas. DGPS measurements were made at various times of the day for reasons related to the availability of satellites and the variation of the angles of refraction during the day ensuring clarity of the signal. The absence of particular electromagnetic obstacles, which often occur in urban areas, this work has benefited. Therefore, the only obstacle that can affect the signal in this work may be due to the particular angle of refraction or related to the various time zones, or the total obscuration of the signal due to the unfavorable disposition of the trees, which prevent the signal from reaching the instrument, causing temporary interruption of the activity. The measurements were made through the use of a handheld Trimble GeoXH. This gives an accuracy GeoXH Handheld decimeter $(10 \mathrm{~cm})$ with optional external antenna Zephyr, thus eliminating the need for data processing. With Bluetooth wireless technology, the GeoXH handheld offers wireless connection to a Bluetooth enabled mobile phone to be able to access the Internet and receive real- time corrections from a VRS network and data on background maps: there is also the possibility to connect wirelessly to other devices, such as laser range finders and bar code readers Bluetooth enabled. The second phase of work involved the processing of the raw data through the use of specific software (Trimble GPS Pathfinder Office 4.0). Through USB connection the raw data have been incorporated into the program to make the necessary corrections. The raw data were corrected for EGNOS mode, based on all the stations on the European Geostationary Navigation System, in support of the Global System GPS. As can be deduced from the points in the correct mode EGNOS were 4,126. The file relative to the right data is saved (in. COR) to analyze the particular characteristic and its plano-altimetric precision. After analyzing the plan relating to the correct points of the survey, it was decided to classify the species of trees found in the park (Fig. 5). This classification was made with the support of a plan, provided by the managers of the park itself, and thanks to some media sources, in order to know the characteristics of the individual essences. The individual punctual entities were then subdivided into many clusters, each provided with its own Layer, in order to facilitate the understanding and the reading of the plan. This plan was later superimposed on the survey NRTK in order to have a clearer view and schematic of the Park and in order to compare the results obtained by the two works in terms of precision.

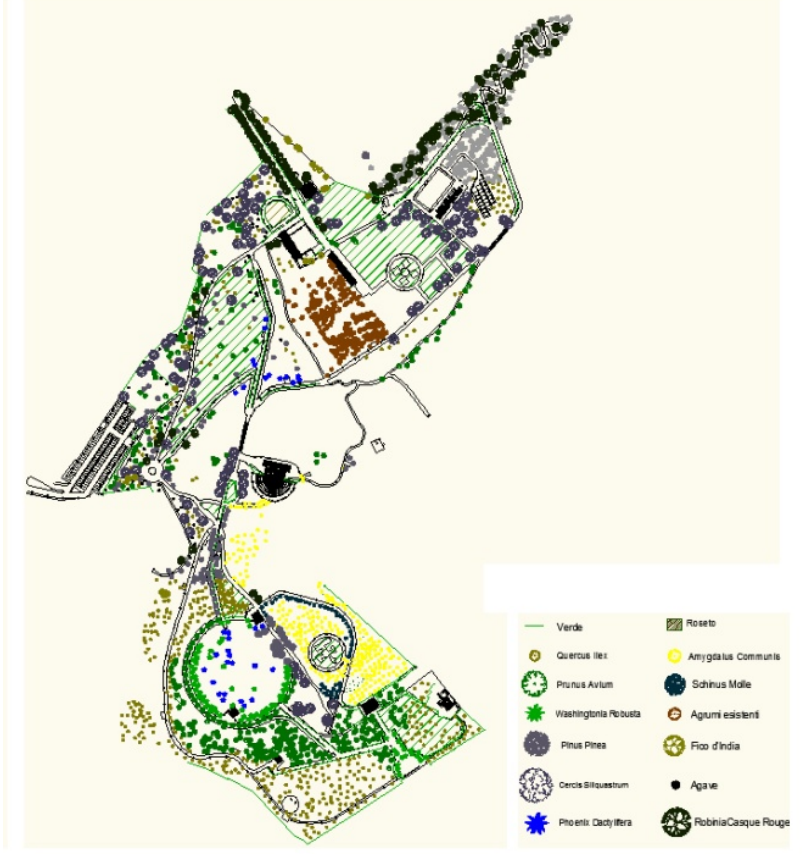

Fig.4: Thematic map of tree species

The last part of the experiment was the study of diagrams useful to define the quality of the field survey, diagrams relating to VDOP, HDOP, ellipsoid and geodetic measurements of the shares and on the measurement accuracy of the altitude and the number of satellites in view. The last processing carried out has focused on the Standard Deviation, which is an index of dispersion of experimental measurements, derived directly from the variance. In particular, $68 \%$ of the measurements should be within the mean-centered and extremes + or - o. In the following diagrams shows the trend of the deviation (in meters) as a function of time over the 5 days. Only a small percentage of data comes out of this range because of the presence of some areas in which the signal was hindered because of the intense presence of trees.Analysis of the results shows that, in respect 
of the index of precision HDOP, $100 \%$ of the values of HDOP is maintained below the value of 5 (the limit beyond which the results are not very accurate and very reliable): in particular, about $90 \%$ of the values stood below the value of 3 . The remaining percentage $(10 \%)$ is between the values 4 and 5 (due to obstacles of various signal reception and also due to a poor coverage of satellites in critical areas of the park).

From the analysis of the values of PDOP emerges that $78 \%$ of the values stood below the value of 3 , while the remaining percentage $(22 \%)$ does not exceed the value of 6 : since the value of PDOP limit fixed by the technical regulations, for ensure that the data have optimal precision is 3 , this data can be considered very satisfactory precision and with more than satisfactory (only a small percentage exceeds this limit of precision, but the results are reliable). As well as for the HDOP, the small percentage of PDOP that is around value between the range 4-6 is given by bad weather conditions during the 5 days of survey and, therefore, from the poor coverage of the satellites because of intense presence of vegetation, which hindered the GPS signal of the instrumentation.

Finally, we studied the percentage distribution of the values (expressed in meters) on Precision Altitude: also on this field has been achieved very satisfactory results since, about $85 \%$ of the values obtained are ranged between 0.1 and 0.5 meters, $9 \%$ is between 1 and 1.5 meters, while the remaining percentage is between 2 and 3 meter: the latter proportion, very negligible, presents a level of precision very poor due to the criticality of the zones detected, in which the dense vegetation has hampered repeatedly receiving the GPS signal of the instrumentation. In general terms, however, the measured data can be considered very reliable. The correction mode EGNOS has allowed to obtain results of high precision even in areas not favorable (for receiving the signal of the receivers) where the intense presence of vegetation has repeatedly generated signal noise. Contrary to expectations, the stage of processing post-processing only a small percentage of values provides indices accuracies next to the technical limit established, while the remaining part of the points detected presents values of PDOP and VDOP very acceptable. The same applies to the Altitude Precision and Standard Deviation for characterize from values very satisfactory and reliable.

\subsection{Mobile Mapping System}

The experimental study was carried out in this work involved the completion of the survey of the park through a system of dynamic detection of TOPCON: The IP-S2. The use of this system mainly affected the surrounding areas the trails in a range of about 30 meters. The preparations were analyzed satellite data such as DOP (Dilution Of Precision), SNR (Signal Noise Ratio), Ionospheric and Multipath errors on the two carriers. Through MMS systems get the precise positioning of the vehicles with the aid of three sensors: a GNSS (Global Navigation Satellite System) dual frequency determines the position in space, an inertial measurement system (Inertial Measurement Unit, IMU) provides the vehicle attitude and a link to the vehicle bus or external wheel encoders (information about the speed of the wheels), allows to obtain odometry information. The IMU which is fitted to the IP-S2 provides information on the acceleration and rotation at high frequency, and if combined with GNSS measurements of precision, allows you to calculate the positions even when you get close to obstacles such as buildings or trees, or while through a tunnel or an overpass. GNSS data can be further processed (postprocessing) for some fine accuracy. The central system processes the sensor data in real time and provides the result, which is given by the fusion of the input data. The file of the recorded data can also be processed in post-processing and filtered off-line, in order to obtain information on the position and the geo-referencing of the data provided by the sensors. Among the features summarized in the data sheet is meant to highlight the instrument accuracy of $\pm 45 \mathrm{~mm}$. We want to emphasize that the degree of accuracy, is closely related to the type of laser scanner integrated in the system and at cruising speed maintained. The complete absence of obstacles and obstruction in the test made it possible to render the entire drive train with a single static initialization, determining the components in the WGS84 reference system. It can be seen that throughout the campaign of acquiring the values of DOP were kept very low, in fact the HDOP is always kept below the value 1.1 except for a small segment, as well as the PDOP and the VDOP you are always kept below the value 2 for the majority of the time; approximately $99 \%$ of the points has values of PDOP less than 3.00, precisely comprised between 1.473 to 3.00 , while the remaining $1 \%$ have values of PDOP greater than 3.00; VDOP for more than $99 \%$ of the points has values between 1.267 to 3.00 , while the remainder has values greater than 3.00. In the three charts peaking is probably due to a sudden lack of visibility given by a satellite obstruction of trees or isolated buildings. You may notice the perfect overlap between the two methods used (RTK and MMS) as seeding points detected always falls within the detected location in RTK mode.

Comparing also the width of the roadway with that measured on the photographic image the difference between the two is still around centimeter. Specifically addressed is the management and processing of laser scanner data acquired. With the aid of suitable software for the processing of point clouds were conducted treatment operations basic laser scanner data, such as noise filtering and sampling, in addition, for experimental purposes, from the point clouds extracted was chosen to study a particular section of the path that has a variation of slope and curvature along its development. We proceeded by analyzing some cross sections in order to analyze their possible level of detail. The data were initially managed using the software SPATIAL FACTORY, owner of Topcon. This data have been displayed and subsequently exported in ASCII format. A first part of the work has been focused in the tracking of the sections along the entire route, in particular, these were plotted in correspondence with the points in which there is a change of curvature and in the intermediate points between the latter so as to be able to trace all 'entire development of the track. In this phase it was also decided to draw the sections perpendicular to the performance of the same route as expected in the case of the realization of a longitudinal profile. Therefore, in addition to the realization of the longitudinal profile has been conducted a study punctual of all sections plotted. From the longitudinal profile has been deduced that the section chosen, which develops about 145 meters, presents a rather regular pattern, in particular it is observed a gradual change of altitude in upward direction. The difference in height between the section to share (101.68 meters) and the section smaller share (96.43 meters) was equal to $5.25 \mathrm{~m}$.

Isolating the individual sections has been possible to observe that the number of points still appears insufficient to outline the performance of the same. Infact these follow a wrong path that does not correspond to real, this is verifiable by the spherical images extracted from the survey (Fig. 5). 


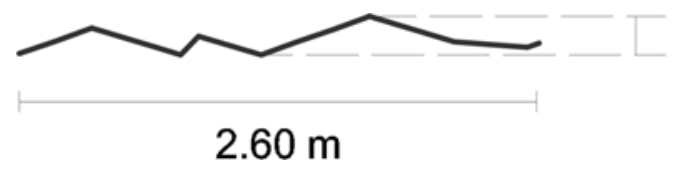

$0.46 \mathrm{~m}$

Fig.5: Cross sections drawn perpendicular to the road axis with automatic processes

Analysis conducted on sections drawn was measured for each of them the difference between the point at higher levels and the one smaller share (in section 1 of 0.66 meters, the first, in section 6 of 0.11 meters the other). The work was therefore continued by placing as its main objective the search for a method that would allow to successfully track sections. From the analysis of the point cloud is observed that the individual points follow a particular trend second parallel strips, and for this, the sections were plotted no longer perpendicular to the development of the path but in parallel to these. To determine the performance of the individual sections, we first carried out by interpolating the points extracted from each section automatically by software (Fig. 6).

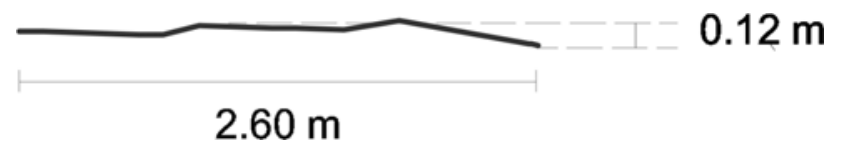

Fig.6: Cross sections extracted along the bands of acquisition with automatic processes

\section{$2.60 \mathrm{~m}$}

Fig.7: Cross sections extracted along the bands of acquisition by manual

Comparing these last sections with the previous one can note that the average performance obtained in this second part is of better quality because the differences in level between the points vary between a minimum of 0,068 meters for the section 2 to a maximum of 0.13 meters for section 5 . It was also decided to draw the sections manually through the plug-in for Rhinoceros Point Tools, and these were represented by more regular and correspondence with the real development of the trail more (Fig. 7).

The average width of the "roadway" is equal to 2.5 meters, the values of the gradients are just inferior results to those obtained in the previous case (the result is greater in correspondence of the section 6 with a value equal to 0.17 meters while the smaller one has in section 4 with a value of 0.033 meters). After we extract the six sections, they were interpolated average change in the contour of the land and then designed a "typical section" idealizing the profile. In the "type section" idealized has been guaranteed a minimum slope in view of the fact that this is a land wrought it will still have its own ability to absorb runoff meteoric. Subsequently were compared the individual cross sections obtained manually in advance with the idealized section; from these comparisons it was observed that the results obtained are of good quality (the value of altitude largest value obtained is equal to about 0.20 meters). In addition we made a "3D model" of the path that was compared with the point cloud in order to gauge the quality of the data acquired during the survey. In this comparison, it was possible to change both the resolution, both the limits of maximum tolerance between the position of the points of the survey and the one of the points of the $3 \mathrm{D}$ model. The precision required in the survey road elements contemplated by Ministerial Decree to populate the database of the road asset refer to DM 1/6/2001, Annex A. Relatively to the precision of the coordinates plano-altimetric regarding the census of the elements in post-processing is expected, by rule, which it assumes a maximum value of $2 \mathrm{~m}$.

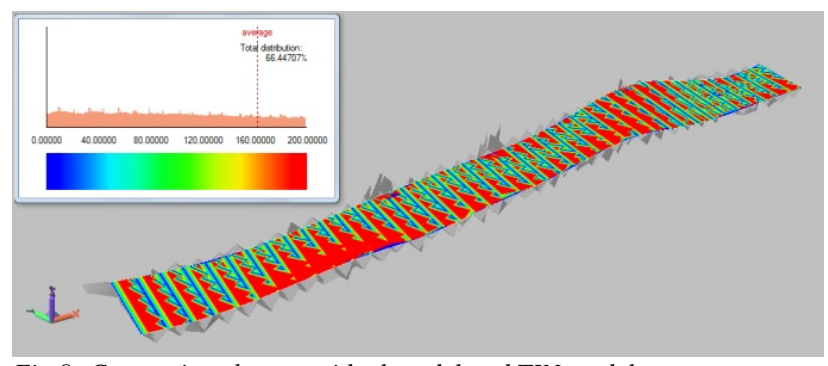

Fig.8: Comparison between ideal model and TIN model

The total distribution of the points surveyed with respect to the ideal model was found to be $85.92 \%$. Even in this case it is possible to observe the particular distribution of points according strips; the overlap between the points of the survey and the ideal model is also in this case more than about $20 \%$ compared to the previous case. Finally the sections obtained manually have been overlying to the path detected in RTK mode in a previous thesis work; between the two surveys, MMS and RTK, was a good match and a good precision of the medium as the sections plotted always fall all 'inside of the detected location in RTK mode. This work has allowed us to carry out an experiment on laser scanner installed on platforms of mobile navigation (equipped with an integrated navigation system GNSS / INS), to carry out a qualitative analysis of the data acquired laser and finally to make the creation of models digital terrain that made it possible to analyze the accuracy of this instrument.

\section{Conclusion}

In conclusion it can be stated that this study has confirmed the possibility of obtaining a survey accurate and detailed also in the case of large urban infrastructure in a short time and at low cost through the integration of methods and techniques as much as possible adapted to respond to individual problems of the work. The experience also confirms the absolute importance to adopt a consistent survey project before activating the phases of acquisition and process data chin. The knowledge of a place, however great, its potential and its problems, the awareness of the ultimate goal of the survey and as a result of the accuracies required, it is necessary condition for the planned use and integration of particular methods and techniques for the acquisition of data that can also be programmed as a function of sub-strategic objectives (size, quantity, location of the objects to be detected). In this case the technologies used all refer to the broad category of GNSS positioning (taking advantage of the vastness of the park surface, the absence of significant blockages and the proximity of the permanent stations of UNIPA, but differ when different becomes the target to reach. A survey in kinematic mode NRTK has proved suitable for the accurate definition of the general elements of the park and fast and light in the acquisition phase and post-processing, the use of a handheld low cost with EGNOS correction has enabled the acquisition of a considerable amount of data in a very short time of five days and a streamlined post processing for the correction of these, ensuring accurate data but definitely less 
well below the tolerances of scale required for the inclusion mapping of an urban setting. The MMS has finally allowed a single day of acquisition, accompanied by a more difficult postprocessing, to incorporate the survey with a huge amount of data and geo-referenced three-dimensional spherical panoramas that have allowed us to:

- Effectual feedback on the website also to the large scale it to define criteria for the control and maintenance of the trails in the park is composed.

- Carefully study the quality of the data allowing a laser reflection on the real ability of this descriptive detail can be deduced by analyzing the extract with both automatic and manual procedures.

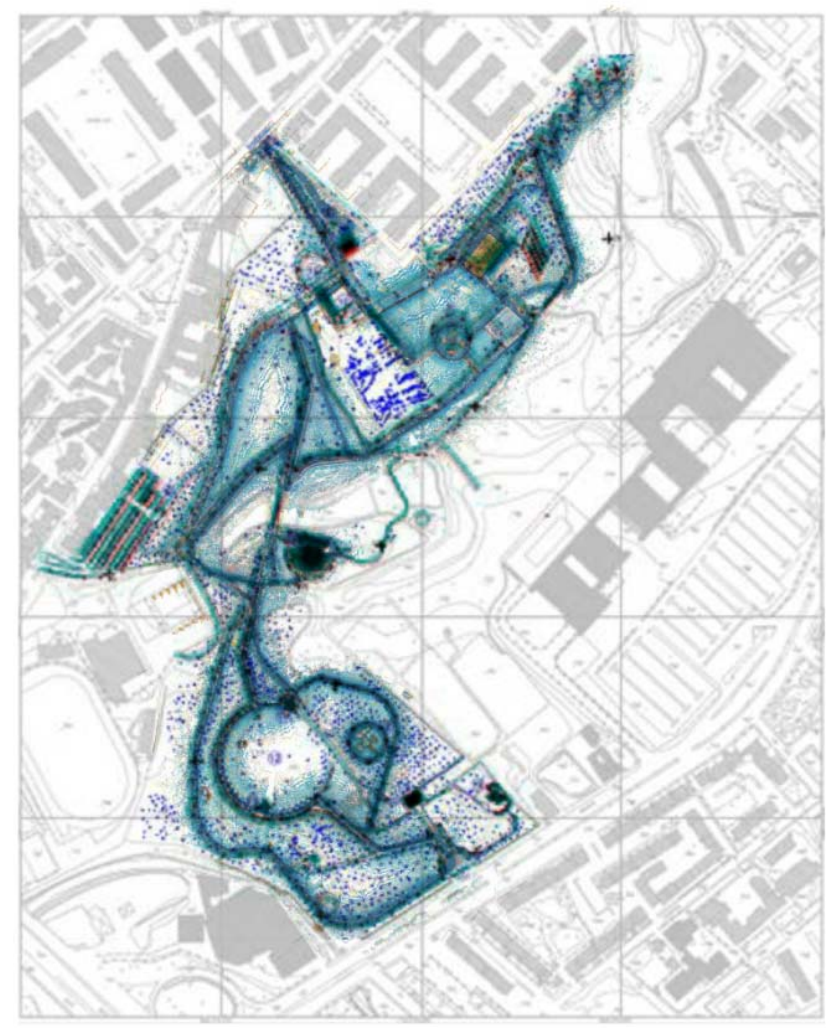

Fig.9: Final step of the work

\section{References}

A. Adami, C. Balletti, F. Guerra, S. Orlandini, P. Vernier (2011) Mobile laser scanning lungo i fronti veneziani del Canal Grande, in Atti del convegnoAsita 2011, Colorno, Parma.

Aponte J, Meng X, Hill C, Moore T, Burbidge M, Dodson A (2009) Quality assessment of a network-based RTK GPS service in the UK. Journal of Applied Geodesy, n.3:25-34

Cefalo R., Gatti M., Sluga T. (2002) Applicazione dei dati di correzione WADGPS da satellite geostazionario EGNOS, in: proceedings of $6^{\circ}$ Conferenza Nazionale ASITA. GEOMATICA per l'Ambiente, il Territorio e il Patrimonio Culturale, 5 - 8 november, Perugina, Italy

Dardanelli G, Franco V, Lo Brutto M (2009) Accuracy and reliability in GNSS NRTK. In: Proceedings of European Navigation Conference - Global Navigation Satellite Systems, Naples, Italy
G. Dondi, A. Simone, C. Lantieri, V. Vignali. (2010). Characterization of pavement surface texture using 3D Laser scanner technique. Proceedings of the 11th International Conference on Asphalt Pavement. August 1-6 2010, Nagoya, Giappone.

Ellum, C.M. and N. El-Sheimy (2001), A mobile mapping system for the survey community, Proceedings of The 3rd International Symposium on Mobile Mapping Technology (MMS 2001). Cario, Egypt

Grejner-Brzezinska D, Kashani I, Wielgosz P (2005) On accuracy and reliability of instantaneous network RTK as a function of network geometry, station separation, and data processing strategy. GPS Solution, n.9:212-225

Lachapelle G, Alves P, Ahn YW (2003) The Effects of Network Geometry on Network RTK Using Simulated GPS Data. In: Proceedings of ION GPS/GNSS 2003, Portland, USA, pp. $1417-1427$

Oszczak S., Ciećko A. Manzoni G., Dziewicki M. Grzegorzewski M., Balint J. (2005). Results of EGNOS/ESTB Marine, Land and Aircraft Experiments in CEEC Region, in: Conference ProDDaGE: DGPS, EGNOS na Wielkich Jeziorach Mazurskich - 24-25 October 2005, Mrągowo, Poland

Porporato C., Cina A., Manzino A. (2007). Applicabilità e attendibilità di ricevitori basso costo per scopi cinematici con l'utilizzo di correzioni di rete, in proceedings: $11^{\circ}$ Conferenza Nazionale ASITA, Centro Congressi Lingotto, Torino $6-9$ november

Schwarz, K.P., Cannon, M.E., and Wong, R.V.C. (1989), A Comparison of GPS Kinematic Models for the Determination of Position and Velocity along a Trajectory, Manuscripta Geodaetica

Schwarz, K.P. and El-Sheimy, N., (1996), Kinematic Multisensor Systems For Close Range Digital Mapping, ISPRS 1996, Commission V, Working Group III, Vienna, Austria

Siligato G., Mussumeci G., (2004), Mobile Mapping System per il CatastoStrade, Dottorato in Ingegneria delle Infrastrutture Viarie Ciclo XIX, Catania

Zhang $\mathrm{K}, \mathrm{Wu} \mathrm{F}, \mathrm{Wu} \mathrm{S}$, Rizos C, Roberts C, Ge L, Yan T, Gordini C, Kealy A, Hale M, Ramm P, Asmussen H, Kinlyside D, Harcombe P (2006) Sparse or dense: challenges of Australian network RTK. In: International Global Navigation Satellite Systems Society IGNSS Symposium 2006, Australia

\section{Acknowledgements}

This work has been supported by many students of University of Palermo. The author wish to acknowledge: Francesco Acuto, Antonino Scozzaro, Matteo Vancheri and Noemi Pucci for cooperation during survey.The authors would also like to thank to the Reviewer for his/her helpful comments and suggestions that substantially improved the manuscript for publication. 